\title{
Dynamic Behavior of a Nonlinear Macro-financial System
}

Azadeh Aram,

Bachelors in Economics, York University

A thesis

presented to Ryerson University

in partial fulfilment of the requirements for the degree of

Master of Science

in the Program of

Applied Mathematics

Toronto, Ontario, Canada

(C)Azadeh Aram

2014 


\section{AUTHOR'S DECLARATION FOR ELECTRONIC SUBMISSION OF A THESIS}

I hereby declare that I am the sole author of this thesis. This is a true copy of the thesis, including any required final revisions, as accepted by my examiners.

I authorize Ryerson University to lend this thesis to other institutions or individuals for the purpose of scholarly research.

I further authorize Ryerson University to reproduce this thesis by photocopying or by other means, in total or in part, at the request of other institutions or individuals for the purpose of scholarly research.

I understand that my thesis may be made electronically available to the public. 


\author{
Abstract \\ Dynamic Behavior of a Nonlinear Macro-financial System \\ Master of Science 2014 \\ Azadeh Aram \\ Applied Mathematics \\ Ryerson University
}

We investigate dynamic behavior of the macro-financial models governed by a system of three first order differential equation involving interest rate, price exponent and investment demand. Using this mathematical model, all the possible behavior that a model shows in the operation of macro-financial system were examined, such as equilibria, stability and Hopf-bifurcations. We find out the ranges of parameters involved in the system under which the equilibria exist the relationship between the parameters and Hopf-bifurcation. Due to changes in conditions in parameters in this financial system, all the behavior of the model as well as the effects of adjustment of the macro-economic policies and adjustment of some parameter on the whole financial system behavior were discussed by applying Ruth-Hurwitz theorem. Hence, It provides better understanding of the lever function of all types of financial policies. 


\section{Acknowledgments}

I would like to express my gratitude to my supervisor Dr. Kunquan Lan, for his excellent guidance, caring, patience, and providing me with an excellent atmosphere for doing research. The help and guidance given by him time to time shall carry me to shape my interest and ideas.

I would like to thank the members of examining committee, Dr. Pawel Pralat, Dr. Silvana llie and Dr. Jean-Paul Pascal for their support and the precious comments for reviewing my thesis. I am obliged to staff members of Ryerson, for the valuable information provided by them in their respective fields. I am grateful for their cooperation during the period of my assignment.

Above all, I thank, my parents, my brother and my friends for their constant encouragement without whom I could not have made it here 


\section{Contents}

1 Introduction 1

1.1 Background . . . . . . . . . . . . . . . . . . . . 1

1.2 Chaotic Systems . . . . . . . . . . . . . . . . . . . 3

1.3 Hopf Bifurcation . . . . . . . . . . . . . . . 4

$\begin{array}{llr}2 & \text { Preliminary } & 7\end{array}$

2.1 Nonlinear Ordinary Differential Equations . . . . . . . . . . . . 7

2.1.1 Basic Definitions and Notations . . . . . . . . . . . . 7

2.2 Local Stability in nonlinear systems of three equations . . . . . . . 10

2.3 Local asymptotic stability of a System . . . . . . . . . . . . . . . 13

2.4 Nonlinear Differential Equations . . . . . . . . . . . . . . . . 14

2.4.1 Smooth Function . . . . . . . . . . . . . . . . . . 14

2.4.2 Routh Hurwitz Theorem . . . . . . . . . . . . . . . . . 16

2.5 Global Stability Vs. Local Stability . . . . . . . . . . . . . . . . . 19

$\begin{array}{llr}3 & \text { Logistic Model } & 20\end{array}$

3.1 Equilibria of the System . . . . . . . . . . . . . . . 21

3.2 Unstability of the system . . . . . . . . . . . . . . . 25

3.3 Local asymptotic stability of the system . . . . . . . . . . . 32

4 Numerical Simulation $\quad 37$

5 Conclusion $\quad 39$

$\begin{array}{lll}6 & \text { Bibliography } & 40\end{array}$

$\begin{array}{ll}\text { Bibliography } & 40\end{array}$ 


\section{Introduction}

\section{$1.1 \quad$ Background}

The Hopf bifurcation of the system is discussed by a rigorous mathematical analysis. The existence of the topological horseshoe in this system is proved by a computer-assisted method. The results obtained in this research would benefit the further studies of this finance chaotic system [18, 17].

Since the discovery of the first classical chaotic in 1963 [1], chaos, as a most fascinating phenomenon in nonlinear dynamical system, has been intensively studied over the past few decades. First we find the chaotic behavior in economic system was first in 1985. In economics the foresight of this behavior meaning that the economic system has an intrinsic indefiniteness. Therefore the study of the finance chaotic system has important value for the stability of economic growth [9].

Liu pointed out that some complicated processes in financial markets needed more in-depth analysis and he studied Hopf bifurcation of a chaotic macroeconomic model. In literature the dynamical behavior and slow manifold of a nonlinear finance chaotic system were investigated [18].

In the theoretical research of the chaotic dynamical systems, Hopf bifurcation analysis plays an important role. Some chaotic systems have been successfully studied by this approach . The horseshoe theory, rigorous but easy to understand for non-mathematicians, is another powerful tool for analyzing chaotic dynamics. Another important result is obtained by Yang which presented a framework on existence of topological horseshoe in non-continuous map. Yangs method has been used successfully in some chaotic neural networks [26].

In finance, stocks and social economics, all types of economic problems are getting 
more and more complicated, due to the influence of nonlinear factors. The evolutive process changing from low dimension to high dimensions causes the variety and complexity of these systems. Sometimes the inappropriate mixture of parameters in economy system causes market to operate improperly, so the market will be out of control as well as many result in financial crisis. Hence doing a systematic and deep study of the internal structure characteristics of such complicated financial system getting more and more important in order to reveal why such complicated phenomena occur, and to predict and dominate the complex continuous financial systems $[8,17]$.

\section{Statement of problems}

The main objective of this study is to investigate how the parameters of the financial system such as saving amount, per-investment cost and elasticity of demands of commercials influence the dynamics of the system. The specific objectives are classified into the following:

i. Analyse the impact of equilibrium of the following system as interest rate, investment demand, and price exponent vary.

ii. Analyse the various topological structures such as, equilibria, zero-growth isocline and limit cycles of Hopf Bifurcation system.

iii. Determine the conditions under which Hopf bifurcation occurs.

\section{Objective of study}

In this financial model, our main goal is first to find the equilibria in order to examine whether the society could afford further cost from misassigned resources. 
The objective of this study is to systematically study the dynamical properties of the nonlinear macro-financial system and clarify how the change in the combination of parameters such as saving amount, per-investment cost and elasticity of demands of commercials can effect the dynamics of the system. We present the bifurcation analysis and it is observed that a proper macro-economic policy and the knowledge of eco-financial characteristic of interacting species should be used in tackling overexploitation or extinction of species.

\section{Methodology}

This complicated financial model contains three nonlinear higher order differential equations. After figuring out the equilibria of the system we need to determine the stability of the model in different range by using Ruth-Hurwitz Theorem. Moreover, we use linearization technique to construct Jacobean Matrix and examine the effect of saving amount, cost of pre-investment and demand elasticity on the market which contain some graphs plotted in MATLAB.

\subsection{Chaotic Systems}

Chaotic research is one of the most remarkable attainment in nonlinear science. Since the chaotic phenomena was first found in 1985, chaotic theories have explained many phenomena, rules, and their inherent complexity, and are revealing the new ones continuously [18]. In the definite system, chaotic known as one of inherent randomness which is caused by internal factor and not by external disturbance which makes them unique $[6,17]$. 
Chaotic systems are complicated systems which are classified into deterministic dynamical systems. They are investigated and applied in a lot of fields for control or forecasting. Deterministic chaos has been severely and broadly analyzed by mathematicians and other scientists. The possibility of giving a precise mathematical definition of deterministic chaos that encapsulates everything in the diverse literature is almost zero. It is in our interest to understand how the idea of chaotic systems is viewed, analysed and used in different fields [22].

At the beginning, it is clear that these systems are defined by strong nonlinearity and by an invariant distribution function and their orbits evolve inside an attractor in which it is possible to do forecasts. Working inside an attractor also permits to control the system and to be able to avoid explosions and strong volatility, which is an interesting task for applications [22].

Now the question is, why are some statisticians so interested by the vast potential which can be gained analyzing deterministic chaos? The main reason is that deterministic dynamical systems can generate chaos, that is highly unpredictable behavior remindful of understanding of random process. In statistics, randomness is generated through a stochastic process and we discuss the stochastic dynamical systems $[22]$.

\subsection{Hopf Bifurcation}

A bifurcation in a financial or economic system is defined as a point or threshold where the system is restructured to operate at a more acceptable or stable level of disequilibrium. Bifurcations do not usually lead to equilibrium conditions, only to a stable or comfortable disequilibrium condition under which the system can continue to survive [19]. 
For a financial system, there can be disequilibrium thresholds where society decides it cannot afford the increasing cost of misallocated resources as disequilibrium increases. Such a threshold then forces a restructuring of the market system. This concept of restructuring to maintain the survival of the system is known as bifurcation theory [2].

Bifurcation is a class of phenomena in dynamic systems such that the dynamic properties of the system cause a sudden qualitative or topological change when the parameter values (the bifurcation parameters) cross a boundary. Bifurcation boundaries, for example, Hopf bifurcations, have been discovered in many macroeconomic systems $[4,5]$.

Bifurcation as a scientific terminology, describes the importance of qualitative changes which happen in the solution curves of a dynamical system, as the key system parameters are varied. It is been used to describe the qualitative stability changes of the solution curves of a nonlinear dynamical system [23].

The study of the finance chaotic system has a meaningful value for the stable economic growth such that some complex processes in financial markets needed more in-depth analysis. In literature, the dynamical nature and slow manifold of a nonlinear finance chaotic system were analysed [21].

Hopf bifurcation analysis represent a significant role in the theoretical research of the chaotic dynamical systems. Some chaotic systems have been successfully studied by this approach [21].

In a differential system when a parameter could vary, the dynamics of the system may alternate. An equilibrium could become unstable and a perodic solution may appear. Also we might have a new stable equilibrium which leads to make previous equilibrium unstable. The value of the parameter at which these changes 
occur is known as a bifurcation value and the parameter that is varied is known as the bifurcation parameter [21].

Hopf Bifurcation or Poincare-Andronov-Hopf Bifurcation is considered as one of the most common type of bifurcation which is theorized by contributions of a French mathematician Jules Henri Poincare (1854-1912), Russian mathematician Alexander A. Andronov (1901-1952), and German mathematician Heinz Hopf (1894-1971) [10].

The Hopf Bifurcation Theorem maintains sufficient conditions for the existing of periodic solutions. The dynamics of the system change from a stable spiral to a centre to an unstable spiral when one parameter is varied, . The eigenvalues of the linearized system change from having negative real parts to zero real parts to positive real parts. Under certain condition, there exist periodic solutions [10].

Bifurcation as a scientific terminology, describes the importance of qualitative changes which happen in the solution curves of a dynamical system, as the key system parameters are varied. It is been used to describe the qualitative stability changes of the solution curves of a nonlinear dynamical system [10]. 


\section{Preliminary}

\subsection{Nonlinear Ordinary Differential Equations}

\subsubsection{Basic Definitions and Notations}

The higher order differential is converted to the following equation which is,

$$
x^{n}(t)=f\left(x^{n-1}(t), \ldots, x(t)\right),
$$

where $x^{n}(t)=\frac{d^{n} x}{d t^{n}}$ and $f\left(x^{n-1}(t), \ldots, x(t)\right)=f\left(\frac{d^{n-1} x}{d t^{n-1}}, \ldots, x\right)$ to the first order differential equation in order to make the model simpler. The autonomous system of differential equations has the form,

$$
\frac{d F}{d t}=F(X)
$$

where $X=\left(x_{1}, x_{2}, \ldots, x_{n}\right)^{T}, F(X)=\left(f_{1}\left(x_{1}, f_{2}, \ldots, f_{n}\right), \ldots, f_{n}\left(x_{1}, x_{2}, \ldots, x_{n}\right)\right)^{T}$ and $\mathrm{F}$ does not explicitly depend on $\mathrm{t}$.

Also there exists an initial value problem which satisfies (2.2) with a given initial condition, $X\left(t_{0}\right)=X_{0}$. Then with the assumption of existing a unique solution to initial value problem, the interval of existence lies in $\left[t_{o}, \infty\right)$. Theorem (2.1) proves the sufficient conditions on the vector function $\mathrm{f}$ for existence and uniqueness of solutions to initial value problems [1].

Theorem 2.1. Suppose that $F$ and $\frac{\partial f}{\partial x_{i}}, i=1,2, . . n$ are continuous functions of $\left(x_{1}, x_{2}, \ldots, x_{n}\right) \in \mathbb{R}^{3}$. Then a unique solution exists to the initial value problem,

$$
\frac{d X}{d t}=F(X) \quad X\left(t_{0}\right)=X_{0}
$$

for any initial value $X_{0} \in \boldsymbol{R}^{n}[1]$. 
Remark: The unique solution may exist. However, the maximal interval of existence $\left[t_{0}, t\right)$ may be finite, $T<\infty$, unless the solution is bounded. It is described para metrically a curve lying in $\mathbb{R}^{n}[1]$.

Definition 2.1. The curve which is lying in $\mathbb{R}^{n}$ is called trajectory, (path or orbit) of a system. The motion of the solution in $\mathbb{R}^{n}$ is determined by the velocity $\frac{d X}{d t}$. The region $\mathbb{R}^{3}$ is called phase space.

There is an important certainty about unique solutions with initial value problems is that non of any two distinct solutions orbit has intersection. Therefore we have the following result in lemma 2.1 [1].

Lemma 2.1. F and $\frac{\partial f}{\partial x_{i}}$ for $i=1, \ldots, n$ are continuous functions of $\left(x_{1}, \ldots, x_{n}\right)$ on $\boldsymbol{R}^{n}$. Furthermore, assume $X_{1}(t), X_{2}(t)$ are the solutions satisfying the differntial system $\frac{d X}{d t}=F(X)$ with the initial conditions $X_{1}(t)=X_{0}$ and $X_{2}(t)=X_{0}$. Then $X_{2}(t)=X_{1}\left(t-t_{0}\right)[1]$.

In the following definition we introduce on of the important kind of solution which is equilibrium solution.

Definition 2.2. The equilibrium solution which can be steady-state solution, fixed point, or critical point, of the differential equation system 2.2 is a constant solution $\bar{X}$ satisfying,

$$
F(\bar{X})=0 .
$$

The Euclidean distance between two points $X_{1}=\left(x_{11}, \ldots, x_{1 n}\right)^{T}$, and $X_{2}=\left(x_{21}, \ldots, x_{2 n}\right)^{T}$ in $\mathbb{R}^{n}$ is,

$$
\left\|X_{1}-X_{2}\right\|_{2}=\sqrt{\sum_{i=1}^{n}\left(x_{1 i}-x_{2 i}\right)^{2}} .
$$


The following definitions are stated for local stability of an equilibrium solution to the system 2.2 :

Definition 2.3. An equilibrium solution $\bar{X}$ of 2.2 is said to be locally stable if for each $\epsilon>0$ there exists a $\delta>0$ with the property that every solution $X(t)$ of the system (2.2) with initial condition $X\left(_{t 0}\right)=X_{0}$, then $\left\|X_{0}-\bar{X}\right\|_{2}<\delta$, satisfies the condition that,

$$
\left\|X_{t}-\bar{X}\right\|_{2}<\epsilon
$$

for all $t \geq t_{0}$ and if the equilibrium solution is not locally stable it is said to be unstable [1].

Definition 2.4. An equilibrium solution $\bar{X}$ is said to be locally asymptotically stable if it is locally stable and if and if there exists $\gamma>0$ such that $\left\|X_{0}-\bar{X}\right\|_{2}<\gamma$ implies

$\lim _{x \rightarrow \infty},\|X(t)-\bar{X}\|_{2}=0$

Now in order to distinguish the local stability and local asymptotic statically equilibrium solution to the system 2.2 , we provide an example.

Example 2.1. Consider the initial value problem $(2.2), X(0)=X_{0}$, where,

$$
B(x, y, z)=\left[\begin{array}{ccc}
b_{11} & 0 & 0 \\
0 & b_{22} & 0 \\
0 & 0 & b_{33}
\end{array}\right]
$$

The origin $\mathrm{X}=0$, is the equilibrium solution to the system $\frac{d X}{d t}=B X$ and the solution $X(t)=(x(t), y(t), z(t))^{T}$ satisfies,

$$
X(t)=x_{0} e^{b_{11} t}\left[\begin{array}{l}
1 \\
0 \\
0
\end{array}\right]+y_{0} e^{b_{22} t}\left[\begin{array}{l}
0 \\
1 \\
0
\end{array}\right]+z_{0} e^{b_{33} t}\left[\begin{array}{l}
0 \\
0 \\
1
\end{array}\right],
$$


where $X_{0}=\left(x_{0}, y_{0}, z_{0}\right)^{T}$.

If $b_{11} \leq 0, b_{22} \leq 0$, and $b_{33} \leq 0$, then $\left\|X_{0}\right\|_{2}<\epsilon$ implies $\left\|X_{t}\right\|_{2}<\epsilon$, and it follows that the zero equilibrium is locally stable.

But now if $b_{11}<0, b_{22}<0$ and $b_{33}<0$ then the zero equilibrium is locally asymptotically stable. Since the solutions converge to zero for any initial value problem, the zero solutions is called globally asymptotically stable. Meaning, The origin is said to be globally asymptotically stable if the whole space $\mathbb{R}^{3}$, is considered as the region of attraction. Moreover, if $b_{i i}>0$ for some $i$, then the zero equilibrium is unstable which can be either a saddle point or unstable node. For instance, if $b_{11}>0$ and $x_{0}>0$ then,

$$
\lim _{t \rightarrow \infty} X(t)=\infty
$$

Then there exists $\epsilon>0$ such that for any $\sigma>0$ and initial condition $\left\|X_{0}\right\|_{2}<\sigma$ with $x_{0}>0,\left\|X_{t}\right\|_{2}>\epsilon$ for some t [1].

Another type of solution is a periodic solution which is limited and continuous and the existence interval is $(-\infty, \infty)$.

Definition 2.5. A periodic solution of the differential system (2.2) is a nonconstant solution $X(t)$ satisfying $X(t+T)=X(t)$ for all t on the interval of existence for some $T>0$. A period of the solution is the minimum value of $T>0$ [1].

Remark: Autonomous scalar equations cannot have periodic solutions. Therefore periodic solutions need two or more differential equations [1].

\subsection{Local Stability in nonlinear systems of three equations}

The local stability of an equilibrium is determined by the eigenvalues of the Jacobean matrix. The functions $f, g$ and $h$ are expanded using Taylor's formula 
about the equilibrium, $\left(x^{*}, y^{*}, z^{*}\right)$, where $u=x-x^{*}, v=y-y^{*}$ and $w=z-z^{*}$. Suppose that $\mathrm{f}, \mathrm{g}$ and $\mathrm{h}$ have continuous second-order partial derivatives in an open set containing the point $\left(x^{*}, y^{*}, z^{*}\right)$. Then,

$$
\begin{aligned}
\frac{d u}{d t}= & f\left(x^{*}+u, y^{*}+v, z^{*}+w\right)=f\left(x^{*}, y^{*}, z^{*}\right)+f_{x}\left(x^{*}, y^{*}, z^{*}\right) u+ \\
& f_{y}\left(x^{*}, y^{*}, z^{*}\right) v+f_{z}\left(x^{*}, y^{*}, z^{*}\right) w+f_{x x}\left(x^{*}, y^{*}, z^{*}\right) \frac{u^{2}}{2}+ \\
& f_{x y}\left(x^{*}, y^{*}, z^{*}\right) u v+f_{x z}\left(x^{*}, y^{*}, z^{*}\right) u w+f_{y y}\left(x^{*}, y^{*}, z^{*}\right) \frac{v^{2}}{2}+\ldots \\
\frac{d u}{d t}= & f\left(x^{*}, y^{*}, z^{*}\right)+f_{x}\left(x^{*}, y^{*}, z^{*}\right) u+ \\
& f_{y}\left(x^{*}, y^{*}, z^{*}\right) v+f_{z}\left(x^{*}, y^{*}, z^{*}\right) w+s_{1}(u, v, w) .
\end{aligned}
$$

where $s_{1}(u, v, w)=f_{x x}\left(x^{*}, y^{*}, z^{*}\right) \frac{u^{2}}{2}+f_{x y}\left(x^{*}, y^{*}, z^{*}\right) u v+$

$$
\begin{aligned}
& f_{x z}\left(x^{*}, y^{*}, z^{*}\right) u w+f_{y y}\left(x^{*}, y^{*}, z^{*}\right) \frac{v^{2}}{2}+\ldots \ldots . \\
& \frac{d v}{d t}= g\left(x^{*}+u, y^{*}+v, z^{*}\right) \\
&= g\left(x^{*}, y^{*}, z^{*}\right)+g_{x}\left(x^{*}, y^{*}, z^{*}\right) u+g_{y}\left(x^{*}, y^{*}, z^{*}\right) v \\
&+g_{x x}\left(x^{*}, y^{*}, z^{*}\right) \frac{u^{2}}{2}+g_{x y}\left(x^{*}, y^{*}, z^{*}\right) u v+g_{y y}\left(x^{*}, y^{*}, z^{*}\right) \frac{v^{2}}{2}+\ldots . . \\
&= g\left(x^{*}, y^{*}, z^{*}\right)+g_{x}\left(x^{*}, y^{*}, z^{*}\right) u+g_{y}\left(x^{*}, y^{*}, z^{*}\right) v+s_{2}(u, v, w) .
\end{aligned}
$$

where $s_{2}(u, v)=g_{x x}\left(x^{*}, y^{*}, z^{*}\right) \frac{u^{2}}{2}+g_{x y}\left(x^{*}, y^{*}, z^{*}\right) u v+g_{y y}\left(x^{*}, y^{*}, z^{*}\right) \frac{v^{2}}{2}+\ldots \ldots$

We assume that $s_{1}$ and $s_{2}$ take small values for small $u, v, w$. In addition, $s_{1}(u, v, w)$ and $s_{2}(u, v, w)$ tend to zero as $u, v$ and $w$ tend to zero. [1] The linearization of the system, obtained by using,

$$
f\left(x^{*}, y^{*}, z^{*}\right)=0, \quad g\left(x^{*}, y^{*}, z^{*}\right)=0, \quad h\left(x^{*}, y^{*}, z^{*}\right)=0,
$$


and neglecting the higher-order terms $s_{1}(u, v, w)$ and $s_{2}(u, v, w)$, is defined to be the three-dimensional linear system,

$$
\begin{aligned}
& \frac{d u}{d t}=f_{x}\left(x^{*}, y^{*}, z^{*}\right) u+f_{y}\left(x^{*}, y^{*}, z^{*}\right) v+f_{z}\left(x^{*}, y^{*}, z^{*}\right) w \\
& \frac{d v}{d t}=g_{x}\left(x^{*}, y^{*}, z^{*}\right) u+g_{y}\left(x^{*}, y^{*}, z^{*}\right) v+g_{z}\left(x^{*}, y^{*}, z^{*}\right) w \\
& \frac{d w}{d t}=h_{x}\left(x^{*}, y^{*}, z^{*}\right) u+h_{y}\left(x^{*}, y^{*}, z^{*}\right) v+h_{z}\left(x^{*}, y^{*}, z^{*}\right) w .
\end{aligned}
$$

Equations (2.10), (2.11) and (2.12) can be expressed in the following vector form,

$$
\left[\begin{array}{c}
\frac{d u}{d t} \\
\frac{d v}{d t} \\
\frac{d w}{d t}
\end{array}\right]=\left[\begin{array}{lll}
f_{x} & f_{y} & f_{z} \\
g_{x} & g_{y} & g_{z} \\
h_{x} & h_{y} & h_{z}
\end{array}\right] \times\left[\begin{array}{c}
u \\
v \\
w
\end{array}\right]
$$

Finally, we can write,

$$
\frac{d Z}{d t}=J Z
$$

where,

$$
\begin{gathered}
Z=\left[\begin{array}{l}
u \\
v \\
w
\end{array}\right] \\
J=\left[\begin{array}{lll}
f_{x} & f_{y} & f_{z} \\
g_{x} & g_{y} & g_{z} \\
h_{x} & h_{y} & h_{z}
\end{array}\right]
\end{gathered}
$$

Local asymptotic stability of the equilibrium depends on the eigenvalues of the Jacobian matrix, which depends on the existence of the partial derivatives in a region containing the equilibrium. Thus for local asymptotic stability of the equilibrium, we need that partial derivatives of $f_{x}, f_{y}$ and $f_{z}$ to be smooth (as we discussed in section 2.4.1) in an open set containing equilibrium. 
The eigenvalues of the Jacobian matrix are the solutions of the characteristic equation.

$$
\operatorname{det}(J-\lambda I)=0
$$

The eigenvalues are the zeros of the following three degree characteristic polynomial:

$$
P(\lambda)=\lambda^{3}+a_{1} \lambda^{2}+a_{2} \lambda+a_{3}
$$

The solution to the non-linear system is characterized by the eigenvalues of the Jacobian matrix J given below. We have used the fact that $f\left(x^{*}, y^{*}, z^{*}\right)=0$ and $g\left(x^{*}, y^{*}, z^{*}\right)=0$. Then the system linearised about the equilibrium $\left(x^{*}, y^{*}, z^{*}\right)$ is $\frac{d Z}{d t}=J Z$, where $Z=(u, v, w)^{T}$ and $\mathrm{J}$ is the Jacobian matrix which is evaluated at the equilibrium:

$$
\begin{gathered}
A(x, y, z)=J(x, y, z)=\left.\left[\begin{array}{lll}
f_{x}(x, y, z) & f_{y}(x, y, z) & f_{z}(x, y, z) \\
g_{x}(x, y, z) & g_{y}(x, y, z) & g_{z}(x, y, z) \\
h_{x}(x, y, z) & h_{y}(x, y, z) & h_{z}(x, y, z)
\end{array}\right]\right|_{x=x^{*}, y=y^{*}, z=z^{*}} \\
J(x, y, z)=\left[\begin{array}{ccc}
f_{x} & f_{y} & f_{z} \\
g_{x} & g_{y} & g_{z} \\
h_{x} & h_{y} & h_{z}
\end{array}\right]
\end{gathered}
$$

such that $f_{x}=\frac{\partial f}{\partial x}, f_{y}=\frac{\partial f}{\partial y}$ and $f_{z}=\frac{\partial f}{\partial z}$, which is evaluated at the equilibrium point $\left(x^{*}, y^{*}, z^{*}\right)[1]$.

\subsection{Local asymptotic stability of a System}

Moiola [10] considered a three order non linear differential equation which has a solution equal to zero. If all of the initial conditions are zero, then this is the 
unique solution to the initial value problem. Then we can determine that the zero solution is "stable", meaning a solution to the initial value problem will tend to zero. Stability of the zero solution depends on the eigenvalues, the roots $\lambda_{i}$, $i=1,2,3$ of the characteristic equation $P(\lambda)=0$. Since solutions have the form $e^{\lambda_{i} t}$, we conclude that solutions to the initial value problems tend to zero when $\lambda_{i}$ are negative numbers or have negative real parts for complex numbers [10].

Solutions to the nonlinear differential equations tends to zero if the eigenvalues $\lambda_{i}$ are negative or have negative real parts, $\lambda_{i}<0$ or $u \pm i v, u<0$. Theorem (2.2) shows that solutions approach zero at an exponential rate if the eigenvalues lie in the left half of the complex plane.

Theorem 2.2. [1] If all of the roots of the characteristic polynomial $P(\lambda)$ are negative or have negative real parts, then given any solution $x(t)$ of the homogeneous differential equation, there exist positive constants $M$ and $b$ such that,

$$
|x(t)| \leq M e^{-b t} \text { for } t>0
$$

and

$$
\lim _{t \rightarrow \infty},|X(t)|=0
$$

Definition 2.6. A function $\mathrm{f}(\mathrm{x})$ is homogeneous of degree $n$, if there exists a constant parameter such as $\lambda$, replacing the variable $\mathrm{x}$ with $\lambda x$ we find:

$$
f(\lambda x)=\lambda^{n} f(x) .
$$

\subsection{Nonlinear Differential Equations}

\subsubsection{Smooth Function}

Firstly we start this chapter by defining a smooth function.

Semmes [24] defines a smooth function by letting $U$ be a nonempty open set in 
$\mathbb{R}^{n}$ for some positive integer $n$, and $f(x)$ be a continuous real or complex-valued function on $U$. Moreover, $f$ is said to be continuously differentiable on $U$ if the first partial derivatives,

$$
\frac{\partial F}{\partial x_{1}}, \ldots, \frac{\partial f}{\partial x_{n}}
$$

exist at every point in $U$ and are continuous on $U$. Similarly, if the first partial derivatives of $\mathrm{f}$ are also continuously-differentiable on $U$, then $f$ is said to be twice continuously-differentiable on $U$. If $i$ is a positive integer, and all derivatives of $f$ of order up to and including $i$ exist on $U$ and are continuous on $U$, then $f$ is said to be $i$ times continuously-differentiable on $U$. If derivatives of $\mathrm{f}$ of all orders exist and are continuous on $U$, then we call $f$ infinitely-differentiable on $U$, or smooth. In other words, a function that has derivatives of all orders is called smooth function.

For introduction, we start with a discussion on the three dimensional autonomous system:

$$
\left\{\begin{array}{l}
\frac{d x}{d t}=f(x, y, z), \\
\frac{d y}{d t}=g(x, y, z), \\
\frac{d z}{d t}=h(x, y, z) .
\end{array}\right.
$$

where $f, g$ and $h$ are smooth nonlinear functions as discussed in section 2.4 .1 of the time variable $t$, with given initial value $\left(x_{0}, y_{0}, z_{0}\right)[11]$.

Definition 2.7. All the solution curves known as trajectories or orbits of the system plotted on the $\mathrm{x}-\mathrm{y}-\mathrm{z}$ space are called generalized phase space as compared to the $x-\bar{x}$ phase space of a one-variable dynamical system [10].

The equilibrium point of the system are the solutions that satisfy simultaneously in the three homogeneous equations which are $f=0, g=0$ and $h=0$. 
Definition 2.8. The equilibrium will be stable if all the nearby trajectories of the system ( with different initial conditions) approach this equilibrium. Also the equilibrium is said to be unstable, if they are moving away from it [10].

Definition 2.9. An equilibrium $\left(x^{*}, y^{*}, z^{*}\right)$ is a solution of the equations

$$
\left\{\begin{array}{l}
f\left(x^{*}, y^{*}, z^{*}\right)=0, \\
g\left(x^{*}, y^{*}, z^{*}\right)=0, \\
h\left(x^{*}, y^{*}, z^{*}\right)=0 .
\end{array}\right.
$$

Thus an equilibrium of (2.19) is a constant solution (or critical points) of the system of differential equations [1].

\subsubsection{Routh Hurwitz Theorem}

Routh-Hurwitz Criteria is one of the essential tools which gives sufficient and necessary conditions for all the roots of the characteristic polynomial with real coefficients which lies in the left half of the complex plane. The name refers to E.J. Ruth and A. Hurwitz who contributed to the formulation of these criteria. In 1875 Ruth developed an algorithm to determine the number of roots that lie in the right half of the complex plane. In 1895, Hurwitz, a German mathematician, verified the determinant criteria for roots to lie in the left half of the complex plane $[1]$.

Based on Theorem 2.2 if the roots of the characteristic polynomial lie in the left half of the complex plane, any solution to the linear, homogeneous differential equation converges to zero. The Ruth-Hurwitz criteria is used to determine local asymptotic stability of an equilibrium for nonlinear system of differential equations which is stated in the following theorem [1]. 
Given the polynomial,

$$
P(\lambda)=\lambda^{n}+a_{1} \lambda^{n-1}+\ldots+a_{n-1} \lambda+a_{n}
$$

where the coefficients $a_{1}, a_{2}, a_{3}$ are real constants. Define the $n$ Hurwitz matrices using the coefficients $a_{i}$ of the characteristic polynomial.

$$
\begin{gathered}
H_{1}=\left[\begin{array}{ll}
a_{1}
\end{array}\right], \\
H_{2}=\left[\begin{array}{ll}
a_{1} & 1 \\
a_{3} & a_{2}
\end{array}\right], \\
H_{3}=\left[\begin{array}{lll}
a_{1} & 1 & 0 \\
a_{3} & a_{2} & a_{1} \\
a_{5} & a_{4} & a_{3}
\end{array}\right],
\end{gathered}
$$

and

$$
H_{n}=\left[\begin{array}{cccccc}
a_{1} & 1 & 0 & 0 & \ldots & 0 \\
a_{3} & a_{2} & a_{1} & 1 & \ldots & 0 \\
a_{5} & a_{4} & a_{3} & a_{2} & \ldots & 0 \\
: & : & : & : & : & \\
: & : & : & : & : & \\
a_{2 n-3} & a_{2 n-4} & a_{2 n-5} & a_{2 n-6} & \ldots & a_{n-2} \\
a_{2 n-1} & a_{2 n-2} & a_{2 n-3} & a_{2 n-4} & \ldots & a_{n} \\
0 & 0 & 0 & 0 & \ldots & a_{n}
\end{array}\right]
$$

where $a_{j}=0$ if $j>n$.

Theorem 2.3. All of the roots of the polynomial $P(\lambda)$ are negative or have negative real part if and only if the determinants of all Hurwitz matrices are positive, that is, det $H_{j}>0$ for $j=1,2, . ., n$. 
Remark: When $n=2$ the Ruth-Hurwitz criteria is simplified to: $\operatorname{det} H_{1}=a_{1}>0$ and $\operatorname{det} H_{2}>0$. Therefore we have:

$$
\begin{gathered}
\operatorname{det}\left(H_{2}\right)=\left|\begin{array}{cc}
a_{1} & 1 \\
0 & a_{2}
\end{array}\right| \\
=a_{1} a_{2}>0
\end{gathered}
$$

or $a_{1}>0$ and $a_{2}>0$. For polynomials of degree $n=2,3,4$ and 5 , the RuthHurwitz criteria are summarized. Ruoth-Hurwitz criteria for $n=2,3,4$ and 5 .

Now the following assertions hold:

a) If $n=2$ then we need to have the condition $a_{1}>0$ and $a_{2}>0$ in order for the equilibrium to be locally asymptotically stable.

b) If $\mathrm{n}=3$ then we need to have the condition $a_{1}>0, a_{3}>0$ and $a_{1} a_{2}>a_{3}$ in order for the equilibrium to be locally asymptotically stable.

c) If $\mathrm{n}=4$ then we need to have the condition $a_{1}>0, a_{3}>0, a_{4}>0$ and $a_{1} a_{2} a_{3}>$ $a_{3}^{2}+a_{1}^{2} a_{4}$, in order for the equilibrium to be locally asymptotically stable.

d)If $\mathrm{n}=5$ then we need to have the condition $a_{i}>0, i=1,2,3,4,5, a_{1} a_{2} a_{3}>$ $a_{3}^{2}+a_{1}^{2} a_{4}$, and $\left(a_{1} a_{4}-a_{5}\right)\left(a_{1} a_{2} a_{3}-a_{3}^{2}-a_{1}^{2} a_{4}\right)>a_{5}\left(a_{1} a_{2}-a_{3}\right)^{2}+a_{1} a_{5}^{2}$ in order for the equilibrium to be locally asymptotically stable.

Remark 2.1. [1] Necessary but not sufficient conditions for the roots of polynomial $P(\lambda)$ to lie in the left half of the complex plane are that the coefficients of the polynomial be strictly positive. Meaning all of the roots of the polynomial are negative or have negative real part iff the determinants of all Hurwitz are positive.

Theorem 2.4. Suppose $\frac{d X}{d t}=F(X)$ is a nonlinear first-order autonomous system with an equilibrium $\bar{X}$. Denote the Jacobean matrix of $F$ evaluated at $\bar{X}$ as $J(\bar{X})$. If the characteristic equation of the Jacobian matrix $J(\bar{X})$, 


$$
\lambda^{3}+a_{1} \lambda^{2}+a_{2} \lambda+a_{3}=0
$$

satisfies the condition of the Routh-Hurwitz criteria in Theorem 2.3, meaning, the determinants of all of the Hurwitz matrices are positive, $\operatorname{det}\left(H_{j}\right)>0, j=1,2,3$, then the equilibrium $\bar{X}$ is locally asymptotically stable. If $\operatorname{det}\left(H_{j}\right)<0$ for some $j=1,2,3$ then the equilibrium $\bar{X}$ is unstable [1].

\subsection{Global Stability Vs. Local Stability}

It is important to distinguish between local and global stability in the nonlinear differential equation. When we have the zero solution it will be considered as equilibrium. The system will approach zero if the zero solution is stable. But, if a positive solution is an equilibrium solution and it is stable, then for initial values close to this equilibrium, solutions approach it.

It is important to distinguish between local and global stability. Local stability of an equilibrium implies that solutions approach the equilibrium only if they are initially close to it. By contrast, global stability of an equilibrium is much stronger and it implies regardless of the initial value, the solutions approach the equilibrium $[1]$. 


\section{Logistic Model}

In this chapter we introduce a complex nonlinear financial model which explains the time variations of three variables: $x$ represents the interest rate, variable $y$ represents investment demand and variable $z$ represents the price exponent. Since sensitivity is of interest, the mentioned variables' changing about time are named of as three new state variables namely: $\dot{x}=\frac{d x}{d t}, \dot{y}=\frac{d y}{d t}$ and $\dot{z}=\frac{d z}{d t}$. Now we discuss the arrangement of the key structures with these state variables [9]. Ma and Chen [9] came up with two main factors that affect the interest rate: first, the surplus between the investment and savings or the contradiction form the investment market influence the interest rate; second, the structure adjustment from goods prices. Thus the change in interest rate is expressed in the following form:

$$
\dot{x}=h_{1}(y-S) x+h_{2} z
$$

where $S$ is the savings amount and $h_{1}$ and $h_{2}$ are constants.

Ma and Chen [9] found that the rate of change of $y, \dot{y}$ is proportional to the rate of investment. Moreover it is inverse proportional to the cost of investment and the interest rate. Therefore:

$$
\dot{y}=h_{3}\left(B-\alpha-\beta x^{2}\right)
$$

where $B$ is the benefit rate of investment, $h_{3}, \alpha$ and $\beta$ are constants.

We can see that changes of $\mathrm{z}$ are dominant by the inconsistency of supply and demand of the commercial market, inflation rate. Moreover, they are controlled by the inflation rate. Suppose the amount of supplies and demands of commercial market is constant in a particular amount of time. Furthermore, commercial market's supplies and demands are in proportion by the price inverse. Yet, the 
changes of inflation rate is stated by the change of real interest rate. It is obvious that the inflation rate equals the nominal interest rate subtracts the real interest rate [9]. Thus, we have the following:

$$
\dot{z}=-h_{4} z-h_{5} x
$$

where $h_{4}$ and $h_{5}$ are both constants.

Huang and Li [17] simplified (3.1), (3.2) and (3.3) in order to obtain the following chaotic financial model which has only three main components $x, y$ and $z$,

$$
\left\{\begin{array}{l}
\dot{x}=z+(y-a) x \\
\dot{y}=1-b y-x^{2} \\
\dot{z}=-x-c z
\end{array}\right.
$$

where $a \geq 0$ is the saving amount, $b \geq 0$ represents the pre-investment cost, and $c \geq 0$ represents the elasticity of demand of commercials.

\subsection{Equilibria of the System}

Based on (3.4) we have the following three equations. Let us call them $f(x, y, z)$, $\mathrm{g}(\mathrm{x}, \mathrm{y}, \mathrm{z})$ and $\mathrm{h}(\mathrm{x}, \mathrm{y}, \mathrm{z})$, respectively. Therefore we have the following:

$$
\left\{\begin{array}{l}
\dot{x}=z+(y-a) x:=f(x, y, z), \\
\dot{y}=1-b y-x^{2}:=g(x, y, z), \\
\dot{z}=-x-c z:=h(x, y, z),
\end{array}\right.
$$

where $f, g$ and $h$ are smooth functions as we defined in section 2.4.1.

Now we can use 2.9 in order to find the equilibria. Then if we let $f(x, y, z)=0$, 
$g(x, y, z)=0$, and $h(x, y, z)=0$, we have the following equation:

$$
\left\{\begin{array}{l}
z=-(y-a) x \\
x^{2}=1-b y \\
x=-c z
\end{array}\right.
$$

In ordet to find the equilibrium, we need to solve the system (3.6) simultaneously. Therefore we have the following theorems for different amount of savings, preinvestment cost and elasticity demand.

Theorem 3.1. If $a \geq 0, b=0$ and $c=0$, then the system (3.5) has no equilibrium.

Proof. If $a \geq 0, b=0$ and $c=0$, the the system (3.5) becomes:

$$
\left\{\begin{array}{l}
z=-(y-a) x \\
x^{2}=1 \\
x=0
\end{array}\right.
$$

which has no solution. Hence (3.5) has no equilibrium.

Remark 3.1. : For positive saving amount, zero amount of price exponent, no preinvestment cost and no elastisity of demand, no equilibrium exists in the market.

Theorem 3.2. If $a \geq 0, b>0$, and $c=0$ then system (3.5) has an equilibrium $\left(0, \frac{1}{b}, 0\right)$.

Proof. Since $z=0$ then from third equation of the system (3.6), we obtain $x=0$. Solving second equation of (3.6):

$$
y=\frac{1}{b}
$$

Therefore, the equilibrium $\left(0, \frac{1}{b}, 0\right)$ is the equilibrium for this case. 
Remark 3.2. The above result more generalize the result of Ma and Chen [9]. Therefore with no elasticity of demand and no price exponent, the equilibrium of the market is $\left(0, \frac{1}{b}, 0\right)$.

Theorem 3.3. If $a>0,0<b<\frac{c}{1+a c}$ and $c>0$, then (3.5) has two equilibria

$$
\begin{gathered}
\left(x_{2}, y_{2}, z_{2}\right)=\left(-\sqrt{1-a b-\frac{b}{c}}, \frac{1}{c}+a,+\frac{\sqrt{1-a b-\frac{b}{c}}}{c}\right) \\
\left(x_{3}, y_{3}, z_{3}\right)=\left(\sqrt{1-a b-\frac{b}{c}}, \frac{1}{c}+a,-\frac{\sqrt{1-a b-\frac{b}{c}}}{c}\right)
\end{gathered}
$$

Proof. Let $\left(x_{2}, y_{2}, z_{2}\right)$ be an interior equilibrium solution of the system (3.5). Then $\left(x_{2}, y_{2}, z_{2}\right)$ is the solution of the system (3.6). Since $z \neq 0$ and $c \neq 0$ then by solving first and third equation of the system (3.5) it implies:

$$
z=-(y-a)(-c z)
$$

Therefore we have:

$$
y=\frac{1}{c}+a
$$

Now from second equation of (3.5) and (3.12) we have the following:

$$
\frac{1}{c}=\frac{\left(1-x^{2}\right)}{b}-a
$$

Reorganizeing above equation we have:

$$
x^{2}=1-a b-\frac{b}{c}
$$

Now let

$$
\xi=c-b-a b c
$$


This case can be divided into three cases: In order for the system (3.5) to have the equilibrium, we need to have the condition of $\xi>0$.

Therefore,

$$
x= \pm \sqrt{1-a b-\frac{b}{c}},
$$

Based on the second equation of the system (3.5),

$$
y=\frac{1}{c}+a,
$$

And from the third equation of the system,

$$
z=\mp \frac{\sqrt{1-a b-\frac{b}{c}}}{c},
$$

It follows from (3.16), (3.17) and (3.18) that (3.9) and (3.10) holds and it is an equilibrium of (3.5).

Remark 3.3. If the condition $b<\frac{c}{1+a c}$ holds for the saving amount, preinvestment cost and the elasticity of demand of commercials we have the equilibrium (3.6).

Theorem 3.4. If $\xi \leq 0$, then the system (3.6) has a unique equilibrium $\left(0, \frac{1}{b}, 0\right)$. Proof. Since $\xi=c-b-a b c \leq 0$ and $x=\mp \sqrt{\frac{\xi}{c}}$ therefore $\xi$ must be zero, consequently, $\mathrm{x}$ and $\mathrm{z}$ must be zero.

Therefore, we conclude that for different $\xi$ the number of equilibria will change. 


\subsection{Unstability of the system}

Baur and Schulze [20] argued that there is no clear agreement of what is called financial stability. Therefore it is much more convenient to find financial instability rather than financial stability but the term of financial instability is often defined inadequately. furthermore, many definitions are relatively wide either in terms of the conditions of financial stability or with regard to the effects of financial stability. In Padoa [25] Financial stability defined as is a condition where the financial system enable to tolerate shocks without giving way to cumulative processes, which impair the allocation of savings and investments opportunities and the processing of payments in the economy. Therefore in this section we discuss the instability of all the equilibria of the financial system.

Now consider the following financial system

$$
\left\{\begin{array}{l}
f(x, y, z)=z+(y-a) x \\
g(x, y, z)=1-b y-x^{2} \\
h(x, y, z)=-x-c x
\end{array}\right.
$$

To study the phase portraits of (3.19) near the interior equilibrium, we need some knowledge of the qualitative theory on phase portrait of planer system near equilibrium. We denote the Jacobin matrix by $\mathrm{J}(\mathrm{x}, \mathrm{y}, \mathrm{z})$ containing $\mathrm{f}, \mathrm{g}$ and $\mathrm{h}$ at the point $(\mathrm{x}, \mathrm{y}, \mathrm{z})$, that is,

Where $f_{x}=(y-a), f_{y}=x, f_{z}=1$ and $g_{x}=-2 x, g_{y}=-b$,

$g_{z}=0, \quad h_{x}=-1, h_{y}=0, h_{z}=-c$. Now the Jacobin matrix is given by:

$$
J(x, y, z)=\left|\begin{array}{ccc}
y-a & x & 1 \\
-2 x & -b & 0 \\
-1 & 0 & -c
\end{array}\right|
$$


Therefore the characteristic matrix is the following:

$$
\begin{aligned}
P(\lambda) & =|\lambda I-J|=\left|\begin{array}{ccc}
\lambda-y+a & -x & -1 \\
2 x & \lambda+b & 0 \\
1 & 0 & \lambda+c
\end{array}\right| . \\
& =\lambda^{3}+a_{1}\left(x^{*}, y^{*}, z^{*}\right) \lambda^{2}+a_{2}\left(x^{*}, y^{*}, z^{*}\right) \lambda+a_{3}\left(x^{*}, y^{*}, z^{*}\right)=0 .
\end{aligned}
$$

Let $(\lambda I-J)=A(x, y, z)$, therefore the determinant and the trace of $\mathrm{A}$ is respectively, $\operatorname{det}(A(x, y, z))$ and $\operatorname{tr}(A(x, y, z))$.

Therefore,

$$
\begin{aligned}
\operatorname{det}(A(x, y, z))= & (y+b)+(\lambda+c)\left((\lambda-y+a)(\lambda+b)+2 x^{2}\right) \\
= & \lambda^{3}+(b-y+a+c) \lambda^{2} \\
+ & \left(1-b y+a b+2 x^{2}+b c-y c+a c\right) \lambda+\left(b-b c y+a b c+2 x^{2} c\right)
\end{aligned}
$$

Now let

$$
\left\{\begin{array}{l}
a_{1}=(b-y+a+c), \\
a_{2}=\left(1-b y+a b+2 x^{2}+b c-y c+a c\right), \\
a_{3}=\left(b-b c y+a b c+2 x^{2} c\right) .
\end{array}\right.
$$

Now based on Theorems (2.3) and (2.4) we need to local assymptotically stability holds. Therefore,

$$
a_{1}>0, \quad a_{3}>0 \quad \text { and } \quad a_{1} a_{2}>a_{3}
$$

In order to find the instability at the equilibrium point $\left(0, \frac{1}{b}, 0\right)$, we have the following theorems.

Theorem 3.5. Assume that $\left(0, \frac{1}{b}, 0\right)$ in an equilibrium point of (3.19). Then the following assertions hold. 
(i) If $b<1, c \leq \frac{1-b^{2}}{b}$ and $a<\frac{1}{b}-b-c$, then the equilibrium point $\left(0, \frac{1}{b}, 0\right)$ of the system (3.19) is unstable.

(ii) If $a b>1, c>\frac{b}{1-a b}$ and $b \leq \frac{c}{1+a c}$, then the equilibrium point $\left(0, \frac{1}{b}, 0\right)$ of the system (3.19) is unstable.

(iii) If $0<b<1, a \leq \frac{1}{b}-b$ and when $c \rightarrow \infty$, then the equilibrium point $\left(0, \frac{1}{b}, 0\right)$ of the system $(3.19)$ is unstable.

Proof. (i) Using the proof by contradiction, in order to have the local assymptotic stability for the equilibrium, we need to have the following first condition of the Routh-Hurwitz (3.21) which is:

$$
(b-y+a+c)>0
$$

If we apply the equilibrium $\left(0, \frac{1}{b}, 0\right)$ to first condition of the Ruth-Hurwitz Theorem (3.21) then we have:

$$
b-\frac{1}{b}+a+c>0 .
$$

which has contradiction with the assumption, so the equilibrium $\left(0, \frac{1}{b}, 0\right)$ is unstable.

(ii) If $b \leq \frac{c}{1+a c}$ therefore we have:

$$
b(1+a c) \leq c
$$

Reorganizing the above inequality we have:

$$
b-c+a b c \leq 0 \text {. }
$$

But in order to have local assymptotical stability for the equilibrium, we need to have the following second condition of the Routh-Hurwitz (3.21) which is:

$$
b-b c y+a b c+2 c x^{2}>0,
$$


applying the equilibrium $\left(0, \frac{1}{b}, 0\right)$ we have:

$$
b-c+a b c>0,
$$

reorganizing the above inequality we have:

$$
b>\frac{c}{1+a c}
$$

which contradicts with the assumption so we obtain that $\left(0, \frac{1}{b}, 0\right)$ is unstable for $b \in\left(0, \frac{c}{1+a c}\right)$ and $a c \neq-1$.

(iii) Using proof by contradiction,we assume that equilibrium point $\left(0, \frac{1}{b}, 0\right)$ is locally assymptotically stable. Therefore by Routh-Hurwitz third condition (3.21) for local assymptotical stability we have,

$$
(b-y+a+c)\left(1-b y+a b+2 x^{2}+b c-y c+a c\right)>\left(b-b c y+a b c+2 c x^{2}\right),
$$

applying the equilibrium $\left(0, \frac{1}{b}, 0\right)$ on the above inequality we have:

$$
\begin{gathered}
\left(b-\frac{1}{b}+a+c\right)\left(a b+b c-\frac{c}{b}+a c\right)>(b-c+a b c) \\
a b^{2}+b^{2} c-c+2 a b c-a+\frac{c}{b^{2}}+a^{2} b-\frac{2 a c}{b}+a^{2} c+b c^{2}-\frac{c^{2}}{b}+a c^{2}-b>0 \\
c^{2}\left[\frac{-1}{b}+a+b\right]+c\left[b^{2}-1+\frac{1}{b^{2}}-\frac{2 a}{b}+a^{2}\right]+\left[a b^{2}-a+a^{2} b-b\right]>0
\end{gathered}
$$

Now dividing the (3.32) by $c^{2}$ we have:

$$
\left[b-\frac{1}{b}+a\right]+\frac{1}{c}\left[b^{2}-1+\frac{1}{b^{2}}-\frac{2 a}{b}+a^{2}\right]+\frac{1}{c^{2}}\left[a b^{2}-a+a^{2} b-b\right]>0,
$$

when $\lim _{c \rightarrow+\infty} f(x, y, z)=f(\infty)$ meaning $\forall \epsilon>0$ there exist $\sigma>0$ such that for large enough $\sigma,|f(x, y, z)-f(\infty)|<\epsilon$.

Therefore for a large enough c, like $c_{0}$ we have:

$$
b-\frac{1}{b}+a>0
$$


which has contradiction with the initial assumption which is $a \leq \frac{1}{b}-b$ and $0<$ $b<1$ and $c \rightarrow \infty$. Therefore, the equilibrium point $\left(0, \frac{1}{b}, 0\right)$ is unstable when $a \leq \frac{1}{b}-b$.

Remark 3.4. In the previous theorem the unstability of the market at the equilibrium $\left(0, \frac{1}{b}, 0\right)$ is determined by Routh-Hurwitz theorem. We conclude that at those ranges for the savings amount, pre-investment cost and the demand elasticity, the market is unstable.

In the following theorems we are going to determine the instability of the market at the equilibrium point $\left(\sqrt{1-a b-\frac{b}{c}}, \frac{1}{c}+a,-\frac{\sqrt{1-a b-\frac{b}{c}}}{c}\right)$.

Theorem 3.6. Assume that $\left(\sqrt{1-a b-\frac{b}{c}}, \frac{1}{c}+a,-\frac{\sqrt{1-a b-\frac{b}{c}}}{c}\right)$ in an equilibrium point of (3.19). Then the following assertions hold.

(i) If $0<c<1, b<\frac{1-c^{2}}{c}$ and $a>0$, then the equilibrium point $\left(\sqrt{1-a b-\frac{b}{c}}\right.$, $\left.\frac{1}{c}+a,-\frac{\sqrt{1-a b-\frac{b}{c}}}{c}\right)$ is unstable.

(ii) If $a>\frac{c-b}{b c}$ and $c>b>0$, then the equilibrium point $\left(\sqrt{1-a b-\frac{b}{c}}, \frac{1}{c}+a\right.$, $\left.-\frac{\sqrt{1-a b-\frac{b}{c}}}{c}\right)$ is unstable.

(iii) If $c>\sqrt{3}, a>\frac{c^{2}-3}{2 c}$ and $b \rightarrow \infty$ and then the equilibrium point $\left(+\sqrt{1-a b-\frac{b}{c}}\right.$, $\left.\frac{1}{c}+a,-\frac{\sqrt{1-a b-\frac{b}{c}}}{c}\right)$ is unstable.

Proof. (i) According to Routh Hurwitz theorem (2.3) the we have, $a_{1}>0, a_{2}>$ 0 simultaneously with $a_{1} a_{2}>a_{3}$ are necessary and sufficient conditions for the 
equilibrium to be stable. Therefore, if one of the conditions does not hold, the equilibrium is unstable. Hence,from the assumption we have:

$$
\begin{aligned}
& -1<c<1 \text { or }|c|<1, \\
& 1>c^{2} \quad \text { or } 1-c^{2}>0,
\end{aligned}
$$

since $c>0$ we have:

$$
\frac{1-c^{2}}{c}>0
$$

based on assumption, $b<\frac{1-c^{2}}{c}$, therefore,

$$
\begin{gathered}
b-\frac{1}{c}+c<0, \\
{\left[b-\left(\frac{1}{c}+a\right)+a+c<0,\right.}
\end{gathered}
$$

which is $a_{1}<0$ with the equilibrium point, $\left(\sqrt{1-a b-\frac{b}{c}}, \frac{1}{c}+a,-\frac{\sqrt{1-a b-\frac{b}{c}}}{c}\right)$. Since it has contradiction with the first condition of local asymptotic stability, we conclude that this equilibrium is unstable.

(ii) According to Routh Hurwitz theorem (2.3) the we have, $a_{1}>0, a_{3}>$ 0 simultaneously with $a_{1} a_{2}>a_{3}$ are necessary and sufficient conditions for the equilibrium to be stable. Therefore, if one of the conditions does not hold, the equilibrium is unstable. Hence,from the assumption we have:

$$
c-b<a b c
$$

We can reorganize the inequality (3.40) to

$$
b-b-a b c+a b c+2 c-2 b-2 a b c<0 .
$$


Thus we have the following inequality,

$$
b-b c\left(\frac{1}{c}+a\right)+a b c+2 c\left(1-\frac{b}{c}-a b\right)<0,
$$

which is $a_{3}<0$ with the equilibrium point, $\left(\sqrt{1-a b-\frac{b}{c}}, \frac{1}{c}+a,-\frac{\sqrt{1-a b-\frac{b}{c}}}{c}\right)$. Since it has contradiction with the first condition of local asymptotic stability, this equilibrium is unstable.

(iii) Using proof by contradiction, we assume that equilibrium point $\left(\sqrt{1-a b-\frac{b}{c}}\right.$, $\left.\frac{1}{c}+a,-\frac{\sqrt{1-a b-\frac{b}{c}}}{c}\right)$ is locally asymptotically stable. Therefore by Routh-Hurwitz third condition (3.21) for local asymptotic stability we have,

$$
(b-y+a+c)\left(1-b y+a b+2 x^{2}+b c-y c+a c\right)>\left(b-b c y+a b c+2 c x^{2}\right) .
$$

Let

$$
\Gamma=\left(1-b\left(\frac{1}{c}+a\right)+a b+b c+2\left(1-a b-\frac{b}{c}\right)-c\left(\frac{1}{c}+a\right)+a c\right),
$$

applying the equilibrium on this condition we have:

$$
\begin{gathered}
\left(b-\left(\frac{1}{c}+a\right)+a+c\right) \Gamma>\left(b-b c\left(\frac{1}{c}+a\right)+a b c+2 c\left(1-a b-\frac{b}{c}\right)\right), \\
\left(b-\frac{1}{c}+c\right)\left(-\frac{b}{c}+b c+2-2 a b-\frac{2 b}{c}\right)>2 c-2 a b c-2 b, \\
b^{2}\left(\frac{-3}{c}+c-2 a\right)+b\left(\frac{1}{c^{2}}+\frac{2 a}{c}+\frac{2}{c^{2}}+c^{2}\right)-\frac{2}{c}>0 .
\end{gathered}
$$

Since $b>0$, we have:

$$
\left(\frac{-3}{c}+c-2 a\right)+\frac{1}{b}\left(\frac{1}{c^{2}}+\frac{2 a}{c}+\frac{2}{c^{2}}+c^{2}\right)-\frac{2}{c b^{2}}>0 .
$$

Therefore for a large enough $b$ we have:

$$
\frac{-3}{c}+c-2 a>0
$$




$$
\frac{c^{2}-3}{2 c}>a
$$

which has the contradiction with the initial assumption. Therefore the equilibrium $\left(\sqrt{1-a b-\frac{b}{c}}, \frac{1}{c}+a,-\frac{\sqrt{1-a b-\frac{b}{c}}}{c}\right)$ is unstable when $c \in(0, \sqrt{3}), \quad \frac{c^{2}-3}{2 c} \leq a$ and $b>0$.

\subsection{Local asymptotic stability of the system}

In this section, we derive some sufficient conditions for local asymptotic stability of both continuous-time nonlinear finance systems. These results have outstanding applications in the stability analysis of physical system models. We determine our stability results using Routh-Hurwitz 2.3 stability theorems.

Remark 3.5. From the previous theorem we conclude that if the amount of preinvestment is large enough, the third condition of Routh-Hurwitz holds. and from the first and second conditions for local asymptotic stability, we have the above conditions for savings amount and elasticity of demand.

Theorem 3.7. If $a>1, b>\frac{-a}{1-a}$, and when $c \rightarrow \infty$ the equilibrium $\left(0, \frac{1}{b}, 0\right)$ is locally asymptotically stable.

Proof. From the third necessary condition for unstability on Routh-Hurwitz theorem (2.3) we have:

$$
\left(b-\frac{1}{b}+a+c\right)\left(a b+b c-\frac{c}{b}+a c\right)>(b-c+a b c)
$$

dividing both sides of the inequality by $c^{2}$ we have:

$$
\left(\frac{b}{c}-\frac{1}{b c}+\frac{a}{c}+1\right)\left(b+\frac{a b}{c}-\frac{1}{b}+a\right)>\left(\frac{b}{c}-\frac{a}{c}+\frac{a b}{c}\right)
$$


Now we conclude that for a large enough amount of c,

$$
a+b-\frac{1}{b}>0
$$

on the other hand from the second condition of the Routh-Hurwitz theorem 2.4 and 2.3 we have:

$$
b-\frac{1}{b}+a+c>0
$$

therefore,

$$
a>\frac{1}{b}-b-c
$$

Similarly from the first condition of the Routh-Hurwitz theorem 2.4 and 2.3 we have:

$$
\begin{gathered}
b-c+a b c>0, \\
b>\frac{c}{1+a c} .
\end{gathered}
$$

Therefore for from the (3.57) and (3.55) and for large enough amount of b, the equilibium is locally assymptotically stable.

Remark 3.6. From the previous theorem we conclude that if the amount of elasticity demand is large enough, the third condition of Routh-Hurwitz holds. and from the first and second conditions for local assymptotical stability, we have the above conditions for savings amount and pre-investment rate.

In the following theorem we determine the local asymptotic stability of the equilibrium $\left(\sqrt{1-a b-\frac{b}{c}}, \frac{1}{c}+a,-\frac{\sqrt{1-a b-\frac{b}{c}}}{c}\right)$ by the same method.

Theorem 3.8. Suppose $a>\frac{6 c}{c^{3}-4 c^{2}+1}$ and let

$$
\chi=\frac{-\left(\frac{3}{c^{2}}+c^{2}+\frac{2 a}{c}\right)-\left(\sqrt{\left(\frac{9}{c^{4}}+c^{4}+\frac{4 a^{2}}{c^{2}}+14+\frac{12 a}{c^{3}}+4 a c-\frac{16 a}{c}-\frac{24}{c^{2}}\right)}\right.}{2\left(c-2 a-\frac{3}{c}\right)},
$$




$$
\psi=\frac{-\left(\frac{3}{c^{2}}+c^{2}+\frac{2 a}{c}\right)+\left(\sqrt{\left(\frac{9}{c^{4}}+c^{4}+\frac{4 a^{2}}{c^{2}}+14+\frac{12 a}{c^{3}}+4 a c-\frac{16 a}{c}-\frac{24}{c^{2}}\right)}\right.}{2\left(c-2 a-\frac{3}{c}\right)},
$$

and $b \in(-\infty, \chi) \cup(\psi,+\infty)$. Then the equilibrium point $\left(\sqrt{1-a b-\frac{b}{c}}, \frac{1}{c}+a\right.$, $\left.-\frac{\sqrt{1-a b-\frac{b}{c}}}{c}\right)$ is locally asymptotically stable.

Proof. For the proof of this theorem we use proof by contradiction. Therefore we assume that the equilibrium point $\left(\sqrt{1-a b-\frac{b}{c}}, \frac{1}{c}+a,-\frac{\sqrt{1-a b-\frac{b}{c}}}{c}\right)$ is unstable. Thus we have for the regarding equilibrium we have the following condition based on Routh Theorem (2.4) $a_{1} a_{2}<a_{3}$. Therefore we have:

$$
(b-y+a+c)\left(1-y b+a b+2 x^{2}+b c-y c+a c\right)<\left(b-b c y+a b c+2 c x^{2}\right) .
$$

Let $\Omega=\left[1-\left(\frac{1}{c}+a\right) b+a b+2\left(1-\frac{b}{c}-a b\right)+b c-\left(\frac{1}{c}+a\right) c+a c\right]$,

$$
\begin{gathered}
{\left[b-\left(\frac{1}{c}+a\right)+a+c\right] \Omega<\left[b-b c\left(\frac{1}{c}+a\right)+a b c+2 c\left(1-\frac{b}{c}-a b\right)\right]} \\
{\left[b-\frac{1}{c}+c\right]\left[2-\frac{3 b}{c}-2 a b+b c\right]<[2 c-2 b-2 a b c] .}
\end{gathered}
$$

Simplifying the above inequality we have:

$$
b^{2}\left[c-2 a-\frac{3}{c}\right]+b\left[\frac{3}{c^{2}}+c^{2}+\frac{2 a}{c}\right]-\frac{2}{c}<0 .
$$

Now let $Q=\frac{3}{c^{2}}+c^{2}+\frac{2 a}{c}, P=c-2 a-\frac{3}{c}$ and $R=\frac{-2}{c}$, hence the inequality (3.63) will change to the following:

$$
P b^{2}+Q b+R=0 \text {. }
$$

Let $\Delta=\left[\frac{3}{c^{2}}+c^{2}+\frac{2 a}{c}\right]^{2}-4\left(\frac{-2}{c}\right)\left(c-2 a-\frac{3}{c}\right)$. Thus,

$$
\Delta=\frac{9}{c^{4}}+c^{4}+\frac{4 a^{2}}{c^{2}}+14+\frac{12 a}{c^{3}}+4 a c-\frac{16 a}{c}-\frac{24}{c^{2}} .
$$


Therefore,

$$
b_{1,2}=\frac{-\left(\frac{3}{c^{2}}+c^{2}+\frac{2 a}{c}\right) \pm\left(\sqrt{\left(\frac{9}{c^{4}}+c^{4}+\frac{4 a^{2}}{c^{2}}+14+\frac{12 a}{c^{3}}+4 a c-\frac{16 a}{c}-\frac{24}{c^{2}}\right)}\right.}{2\left(c-2 a-\frac{3}{c}\right)},
$$

Let $\chi=\frac{-\left(\frac{3}{c^{2}}+c^{2}+\frac{2 a}{c}\right)-\left(\sqrt{\left(\frac{9}{c^{4}}+c^{4}+\frac{4 a^{2}}{c^{2}}+14+\frac{12 a}{c^{3}}+4 a c-\frac{16 a}{c}-\frac{24}{c^{2}}\right)}\right.}{2\left(c-2 a-\frac{3}{c}\right)}$, and $\psi=\frac{-\left(\frac{3}{c^{2}}+c^{2}+\frac{2 a}{c}\right)+\left(\sqrt{\left(\frac{9}{c^{4}}+c^{4}+\frac{4 a^{2}}{c^{2}}+14+\frac{12 a}{c^{3}}+4 a c-\frac{16 a}{c}-\frac{24}{c^{2}}\right)}\right.}{2\left(c-2 a-\frac{3}{c}\right)}$.

Thus for $b \in(-\infty, \chi) \cup(\psi,+\infty)$, the equilibrium $\left(\sqrt{1-a b-\frac{b}{c}}, \frac{1}{c}+a,-\frac{\sqrt{1-a b-\frac{b}{c}}}{c}\right)$ is locally asymptotically stable.

It is obvious that we have the same condition for the equilibrium $\left(-\sqrt{1-a b-\frac{b}{c}}\right.$, $\left.\frac{1}{c}+a,+\frac{\sqrt{1-a b-\frac{b}{c}}}{c}\right)$.

Theorem 3.9. Suppose $a>0, a b>1$ and $c=0$, then the equilibrium point $\left(0, \frac{1}{b}, 0\right)$ is locally asymptotically stable.

Proof. Since $a b>1, a>0$ and $b>0$, we have the following equation:

$$
a b(a+b)>a+b
$$

Reorganizing the above equation we have:

$$
\begin{gathered}
a b^{2}-a+a^{2} b>b, \\
\left(b-\frac{1}{b}+a\right) a>1 .
\end{gathered}
$$

Finally we obtain: 


$$
\left(b-\frac{1}{b}+a\right)(a b)>b .
$$

Using Theorem 2.4 we have:

$$
a_{1} a_{2}>a_{3}
$$

which is the sufficient condition for local asymptotic stability of the equilibrium $\left(0, \frac{1}{b}, 0\right)$.

Remark 3.7. We conclude that if we let the elasticity of demand of the market equals to zero, the equilibrium point $\left(0, \frac{1}{b}, 0\right)$ is locally asymptotically stable. 


\section{Numerical Simulation}

In this section we notice that the graph will change as we slightly change the parameters a, b and c. Consider the change of elasticity of demand c of the system and keep other parameters fixed. From the previous analysis we can see that the unique equilibrium $\left(0, \frac{1}{b}, 0\right)$ of the system is a stable sink when $(c-b-a b c)<0$ and $b>0$. Here we take $a=4, b=0.125$ and $c=0.5$. Therefore we have the Figure 2.

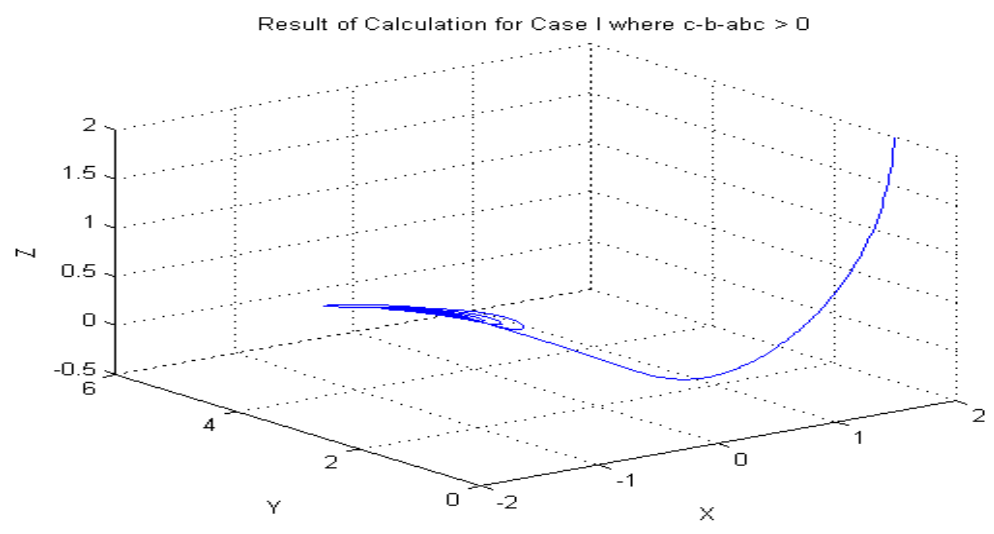

Figure 1: Result of calcultation for case 1 where $c-b-a b c>0$

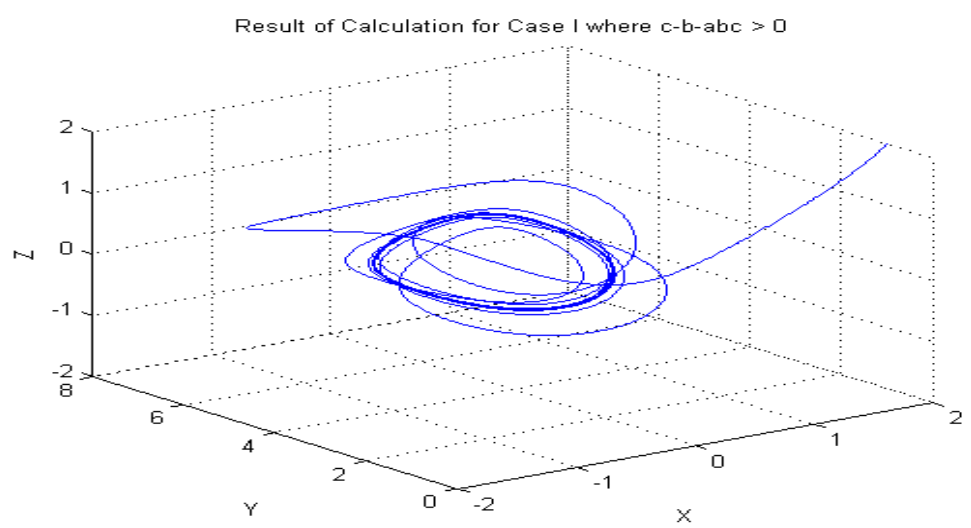

Figure 2: Saddle structure of the system with $\mathrm{a}=4 . \mathrm{b}=0.125, \mathrm{c}=0.5$ 
Now if we decrease the elasticity of demand c from 0.5 to 2.03417452 , we obtain Figure.3. In conclusion, if the macro-finance system (3.4) undergoes a

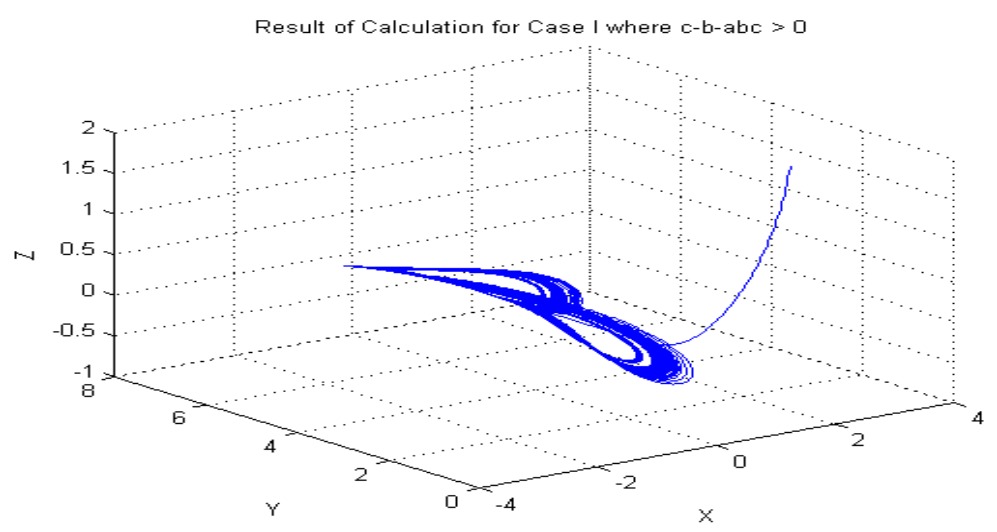

Figure 3: Saddle structure of the system with $\mathrm{a}=4 . \mathrm{b}=0.125, \mathrm{c}=2.03417452$

Hopf bifurcation under some parameters, we should carefully regulate perturbation parameters and the initial values. Therefore, we can maintain the price and keep the society balanced. 


\section{Conclusion}

From the results of the above simulation, we conclude that first, if the combination of the parameters in the system is not appropriate, therefore chaotic occurs in the finance system. Meaning that it is likely for the finance system to lose control and force the system plunged into the stagnant and ossified state. Hence, no matter when it is in the inflation stage or in the economic slack stage, the transformation of the mechanic or the adjustment of the structure will forever be the first task in the reform of financial system [9].

Secondly, the elasticity of variables leads to weaken the information feedback and it can only provide the strategic institute distorted and wrong signals. The bad results caused by this can be estimated that these bad results will continuously extend or expands the form of a vicious cycle. Therefore, strengthening the elasticity of the variables appropriately will help to the stable economy and the normal operation of the financial system [9].

We developed many different results for future research work regarding the complicated nonlinear macro-finance system. Many of the theoretical and simulation technique developed in this work should be useful in pursuing the future research. In our model we have In the future, we plan to investigate the time delay feedback on this financial model. Therefore we are unable to find deep and more accurate result in chaotic behavior. 


\section{Bibliography}

\section{References}

[1] L. J. S. Allen, An introduction to mathematical biology, Pearson Prentice Hall, NJ, (2007).

[2] Baogui Xin,Tong Chen, and Junhai Ma, Neimark-Sacker Bifurcation in a Discrete-Time Financial System, Hindawi Publishing Corporation Discrete Dynamics in Nature and Society, 12 (2010), 405-639.

[3] 1. C.I. Byrnes and V. Sundarapandian, Persistence of equilibria for locally asymptotically stable systems, Int.J. Robust Nonlinear Control 11, (2001), 87-93.

[4] J. Benhabib and K. Nishimura, The Hopf bifurcation and the existence and stability of closed orbits in multisector models of optimal economic growth, Journal of Economic Theory 21, (1997), 421-444.

[5] W. Barnett and Y. He, Bifurcations in macroeconomic models, in Economic Growth and Macroeconomic Dynamics. Recent Developments in Economic Theory, S. Dowrick, P. Rohan, and S. Turnovsky. Cambridge University Press, Cambridge UK, (2004), 95-112.

[6] Cheng Si-wei. Complicated Science and Management [A]. Article Collection of Beijing Xiangshan Conference, Beijing:Science Press, C(1998), 1-9.

[7] Junhai Ma, Yaqiang Cui, Lixia Liu. Hopf Bifurcation and Chaos of Financial System on Condition of Specific Combination of Parameters, Springer Science and Business Media, LLC, 37(2008), 1104-1112. 
[8] Q. Gao and J. Ma, Chaos and Hopf bifurcation of a finance system, Nonlinear Dynamics, 58(2009), 209-216.

[9] Jun-hai Ma, Yu-shu Chen. Study for the Bifurcation Topological Structure and the Global Complicated Character of a kind of Nonlinear Finance System (II),Shanghai University, Shanghai, China, 22(2001)(12).

[10] Jorge L. Moiola, Hopf Bifurcation Analysis, World Scientific Pub Co Inc(199606), 326.

[11] Raymond O’Nail Wells, Defferential Analysis on Complex Manifold,Springers Science, 3(2008).

[12] C. S. Holling, The functional response of predator to prey density, and its role in mimicry and population regulation, Mem. Ent. Soc. Can. 45(1965) 1-60.

[13] A. N. Kolmogorov, Sulla, Teoria di Volterra della Lotta per l'Esisttenza, Giorna. Instituto Ital. Attuarri, 7(1936), 74-80.

[14] K.Q.Lan, C.R.Zhu, Phase portraits, Hopf bifurcations Tanner models for predator-prey interactions, Non-linear Anal.Appl 12(2011) 1961-1973.

[15] K.Q.Lan, C.R.Zhu, Phase portraits, Hopf bifurcations and limit cycles of Leslie-Gower predator-prey systems with harvesting rates, Discrete and continuous dynamical systems 14 (2010) 289-306.

[16] K.Q.Lan, C.R.Zhu, Phase portraits of predator-prey systems with harvesting rates, Discrete and continuous dynamical systems 32(2012), 901-933.

[17] Huang Deng-shi, Li Hong-qing. Theory and Method of the Nonlinear Economics Publishing [M]. Chengdu; House of Sichuan University, (1993). 
[18] Junhai Ma, Yaqiang Cui, Lixia Liu. Hopf Fifurcation and Chaos of Financial System on Condition of Specific Combination of Parameters, Springer Science and Business Media, LLC, (2008).

[19] D. Nawrocki, Entropy, bifurcation and dynamic market disequilibrium,Financial Review,19,(1984), 266-284.

[20] Dirk G.Baur, Niels Schulze, Financial market stabilityA test", Journal of International Financial Markets, Institutions and Money, Elsiver Publication, nt. Fin. Markets, Inst. and Money 19,(2009), 506519.

[21] Fran cois Primeau and David Newman, Elongation and Contraction of the Western Boundary Current Extension in a Shallow-Water Model: a Bifurcation Analysis, Journal of Physical Oceanography 27,(2006).

[22] Gugan D. "Chaos in Economics and Finance", PSE, University Paris1 Panthon -Sorbonne, CES-MSE, 106 Bd de lhopital, (2006),75-113.

[23] Morris W. Hirsch, Stephen Smale and Robert L. Devaney, Differential Equations, Dynamical Systems, and an Introduction to Chaos, Elsevier Academic Press, 60 (2),(2004).

[24] Stephen Semmes, An introduction to some aspects of functional analysis, 5: Smooth functions and distributions, Rice University Publication, (1998).

[25] Padoa-schioppa, Central Banks and Financial Stability, European Central Bank, 2,(2003).

[26] Lan Yueheng, Dynamical system approaches to one-dimensional spatiotemporal chaos, School of physics, Georgia Institute of technology, (2004). 